Pacific Journal of Mathematics

SOME CONGRUENCES FOR THE BELL POLYNOMIALS 


\section{SOME CONGRUENCES FOR THE BELL POLYNOMIMALS}

\section{CARLitz}

1. Let $\alpha_{1}, \alpha_{2}, \alpha_{3}, \cdots$ denote indeterminates. The Bell polynomial $\phi_{n}\left(\alpha_{1}, \alpha_{2}, \alpha_{3}, \cdots\right)$ may be defined by $\phi_{0}=1$ and

$$
\phi_{n}=\phi_{n}\left(\alpha_{1}, \alpha_{2}, \alpha_{3}, \cdots\right)=\sum \frac{n !}{k_{1} !(1 !)^{k_{1}} k_{2} !(2 !)^{k_{2}} \cdots} \alpha_{1}^{k_{1}} \alpha_{2}^{k_{2}} \cdots,
$$

where the summation is over all nonnegative integers $k_{j}$ such that

$$
k_{1}+2 k_{2}+3 k_{3}+\cdots=n \text {. }
$$

For references see Bell [2] and Riordan [5, p. 36]. The general coefficient

$$
A_{n}\left(k_{1}, k_{2}, k_{3}, \cdots\right)=\frac{n !}{k_{1} !(1 !)^{k_{1}} k_{2} !(2 !)^{k_{2}} \cdots}
$$

is integral; this is evident from the representation

$$
A_{n}\left(k_{1}, k_{2}, k_{3}, \cdots\right)=\frac{n !}{k_{1} !\left(2 k_{2}\right) !\left(3 k_{3}\right) ! \cdots} \cdot \frac{\left(2 k_{2}\right) !}{k_{2} !(2 !)^{k_{2}}} \frac{\left(3 k_{3}\right) !}{k_{3} !(3 !)^{k_{3}}} \cdots
$$

and the fact that the quotient

$$
\frac{(r k) !}{k !(r !)^{k}}
$$

is integral [1, p. 57].

The coefficient $A_{n}\left(k_{1}, k_{2}, k_{3}, \cdots\right)$ resembles the multinomial coefficient

$$
M\left(k_{1}, k_{2}, k_{3} \cdots\right)=\frac{\left(k_{1}+k_{2}+k_{3}+\cdots\right) !}{k_{1} ! k_{2} ! k_{3} \cdots} .
$$

If $p$ is a fixed prime it is known [3] that $M\left(k_{1}, k_{2}, k_{3}, \ldots\right)$ is prime to $p$ if and only if

$$
\begin{array}{cc}
k_{i}=\sum_{j} a_{i j} p^{j} & \left(0 \leqq a_{i j}<p\right), \\
k_{1}+k_{2}+k_{3}+\cdots=\sum_{j} a_{j} p^{j} & \left(0 \leqq a_{j}<p\right)
\end{array}
$$

and

$$
\sum_{i} a_{i j}=a_{j} \quad(j=0,1,2, \cdots) .
$$

It does not seem easy to find an analogous result for $A_{n}\left(k_{1}, k_{2}, k_{3}, \cdots\right)$. For some special results see $\S 3$ below. 
Bell [2] showed that

$$
\phi_{p} \equiv \alpha_{1}^{p}+\alpha_{p}
$$

and also determined the residues $(\bmod p)$ of $\phi_{p+1}, \phi_{p+2}, \phi_{p+3}$. He also obtained an expression for the residue of $\phi_{p+r}$ as a determinant of order $r+1$. Generalizing (1.3) we shall show first that

$$
\phi_{p^{r}} \equiv \alpha_{1}^{p^{r}}+\alpha_{p}^{p^{r-1}}+\cdots+\alpha_{p^{r}}
$$

and that

$$
\phi_{p n}\left(\alpha_{1}, \alpha_{2}, \alpha_{3}, \cdots\right) \equiv \phi_{n}\left(\phi_{p}, \alpha_{2 p}, \alpha_{3 p}, \cdots\right)
$$

for all $n \geqq 1$. Note that on the right the first argument in $\phi_{n}$ is $\phi_{p}$ and not $\alpha_{p}$.

2. From (1.1) we get the generating function

$$
\sum_{n=0}^{\infty} \phi_{n} \frac{t^{n}}{n !}=\exp \left(\alpha_{1} t+\alpha_{2} \frac{t^{2}}{2 !}+\alpha_{3} \frac{t^{3}}{3 !}+\cdots\right) \text {. }
$$

Indeed this may be taken as the definition of $\phi_{n}$. Differentiating with respect to $t$ we get

$$
\sum_{n=0}^{\infty} \phi_{n+1} \frac{t^{n}}{n !}=\sum_{n=0}^{\infty} \phi_{n} \frac{t^{n}}{n !} \sum_{r=0}^{\infty} \alpha_{r+1} \frac{t^{r}}{r !},
$$

so that

$$
\phi_{n+1}=\sum_{r=0}^{n}\left(\begin{array}{l}
n \\
r
\end{array}\right) \phi_{n-r} \alpha_{r+1} .
$$

Since the binomial coefficient

$$
\left(\begin{array}{c}
p n \\
r
\end{array}\right) \equiv 0
$$

unless $p \mid r$ and

$$
\left(\begin{array}{l}
p n \\
p r
\end{array}\right) \equiv\left(\begin{array}{l}
n \\
r
\end{array}\right)
$$

it follows from (2.2) that

$$
\phi_{p n+1} \equiv \sum_{r=0}^{n}\left(\begin{array}{l}
n \\
r
\end{array}\right) \phi_{p(n-r)} \alpha_{p r+1} \quad(\bmod p) .
$$

If for brevity we put

$$
A(t)=\sum_{r=1}^{\infty} \alpha_{r} t^{r} / r !,
$$


so that

$$
\sum_{n=0}^{\infty} \phi_{n} \frac{t^{n}}{n !}=\exp A(t)
$$

it is easily seen by repeated differentiation and by (1.3) that

$$
\sum_{n=0}^{\infty} \phi_{n+p} \frac{t^{n}}{n !} \equiv\left\{\left(A^{\prime}(t)\right)^{p}+A^{(p)}(t)\right\} e^{\Lambda(t)}
$$

(By the statement

$$
\sum_{n=0}^{\infty} A_{n} \frac{t^{n}}{n !} \equiv \sum_{n=0}^{\infty} B_{n} \frac{t^{n}}{n !}
$$

where $A_{n}, B_{n}$ are polynomials with integral coefficients, is meant the system of congruences

$$
\left.A_{n} \equiv B_{n} \quad(\bmod m) \quad(n=0,1,2, \cdots)\right) .
$$

Hurwitz [4, p. 345] has proved the lemma that if $a_{1}, a_{2}, a_{3}, \ldots$ are arbitrary integers then

$$
\left(\sum_{n=1}^{\infty} a_{n} \frac{x^{n}}{n !}\right)^{k} \equiv 0
$$

The proof holds without change when the $a_{n}$ are indeterminates. Since

$$
A^{\prime}(t)=\sum_{n=0}^{\infty} \alpha_{n+1} \frac{t^{n}}{n !}
$$

it follows easily from Hurwitz's lemma that

$$
\left(A^{\prime}(t)^{p}=\left(\alpha_{1}+\sum_{n=1}^{\infty} \alpha_{n+1} \frac{t^{n}}{n !}\right)^{p} \equiv \alpha_{1}^{p}\right.
$$

Thus (2.4) becomes

$$
\sum_{n=0}^{\infty} \phi_{n+p} \frac{t^{n}}{n !} \equiv\left(\alpha_{1}^{p}+\sum_{r=0}^{\infty} \alpha_{r+p} \frac{t^{r}}{r !}\right) \sum_{n=0}^{\infty} \phi_{n} \frac{t^{n}}{n !},
$$

which yields

$$
\phi_{n+p} \equiv\left(\alpha_{1}^{p}+\alpha_{p}\right) \phi_{n}+\sum_{r=1}^{n}\left(\begin{array}{l}
n \\
r
\end{array}\right) \alpha_{r+p} \phi_{n-r} \quad(\bmod p) .
$$

In particular, for $n=0,(2.5)$ reduces to Bell's congruence (1.3). Similarly

$$
\begin{gathered}
\phi_{p+1} \equiv\left(\alpha_{1}^{p}+\alpha_{p}\right) \alpha_{1}+\alpha_{p+1} \equiv \phi_{p} \alpha_{1}+\alpha_{p+1} \\
\phi_{p+2} \equiv \phi_{p} \phi_{2}+2 \alpha_{p+1} \alpha_{1}+\alpha_{p+2},
\end{gathered}
$$


and so on.

We remark that (2.5) is equivalent to Bell's congruence involving a determinant [2, p. 267, formula (6.5)]. Also for $s=\alpha_{1}=\alpha_{2}=\cdots,(2.5)$ reduces to

$$
\begin{aligned}
a_{n+p}(s) & \equiv\left(s^{p}+s\right) a_{n}(s)+s \sum_{r=1}^{n}\left(\begin{array}{l}
n \\
r
\end{array}\right) a_{n-r}(s) \\
& \equiv a_{n+1}(s)+s^{p} a_{n}(s)
\end{aligned}
$$

where [5, p. 76]

$$
a_{n}(s)=\phi_{n}(s, s, \cdots)=\sum_{k} S(n, k) s^{k}
$$

and $S(n, k)$ denotes the Stirling number of the second kind. The congruence $(2.5)^{\prime}$ is due to Touchard [6].

If in (2.5) we replace $n$ by $p n$ we get

$$
\phi_{p(n+1)} \equiv \phi_{p} \phi_{n p}+\sum_{r=1}^{n}\left(\begin{array}{l}
n \\
r
\end{array}\right) \alpha_{p(r+1)} \phi_{p(n-r)}
$$

for all $n=0,1,2, \cdots$. Thus $\phi_{p n}$ is congruent to a polynomial in $\phi_{p}$, $\alpha_{2 p}, \alpha_{3 p}, \cdots$ alone. Moreover, comparing (2.6) with (2.2), it is clear that

$$
\phi_{p n} \equiv \phi_{n}\left(\phi_{p}, \alpha_{2 p}, \alpha_{3 p}, \cdots\right)
$$

so that we have proved (1.5).

Replacing $n$ by $p n$ in (2.7) we get

$$
\phi_{p^{2} n} \equiv \phi_{p n}\left(\phi_{p}, \alpha_{2 p}, \alpha_{3 p}, \cdots\right) \equiv \phi_{n}\left(\phi_{p}^{p}+\alpha_{p^{2}}, \alpha_{2 p^{2}}, \alpha_{3 p^{2}}, \cdots\right) \text {. }
$$

In particular for $n=1$

$$
\phi_{p^{2}} \equiv \phi_{p}^{p}+\alpha_{p^{2}} \equiv \alpha_{1}^{p^{2}}+\alpha_{p}^{p}+\alpha_{p^{2}}
$$

Again replacing $n$ by $p n$ we get

$$
\phi_{p^{3} n} \equiv \phi_{n}\left(\phi_{p^{2}}^{p}+\alpha_{p^{3}}, \alpha_{2 p^{3}}, \alpha_{3 p^{3}}, \cdots\right),
$$

so that in particular

$$
\phi_{p^{3}} \equiv \phi_{p^{2}}^{p}+\phi_{p^{3}} \equiv \alpha_{1}^{p^{3}}+\alpha_{p}^{p^{2}}+\alpha_{p^{2}}^{p}+\alpha_{p^{3}}
$$

Continuing in this way we see that

$$
\phi_{p^{r} n} \equiv \phi_{n}\left(\phi_{p^{r}}, \alpha_{2 p^{r}}, \alpha_{3 p^{r}}, \cdots\right)
$$

and

$$
\phi_{p^{r}} \equiv \phi_{p r-1}^{p}+\alpha_{p^{r}} \equiv \alpha_{1}^{p^{r}}+\alpha_{p}^{p^{r-1}}+\cdots+\alpha_{p^{r}} \quad(\bmod p) .
$$

We have therefore proved (1.4) as well as the more general congruence (2.8). 
Since

$$
\begin{aligned}
& \phi_{2}=\alpha_{1}^{2}+\alpha_{2}, \\
& \phi_{3}=\alpha_{1}^{3}+3 \alpha_{1} \alpha_{2}+\alpha_{3}, \\
& \phi_{4}=\alpha_{1}^{4}+6 \alpha_{1}^{2} \alpha_{2}+4 \alpha_{1} \alpha_{3}+3 \alpha_{2}^{2}+\alpha_{4},
\end{aligned}
$$

it follows from (2.8) that

$$
\left\{\begin{array}{l}
\phi_{2 p^{r}} \equiv \phi_{p^{r}}^{2}+\alpha_{2 p^{r}}, \\
\phi_{3 p^{r}} \equiv \phi_{p^{r}}^{3}+3 \phi_{p^{r}} \alpha_{2 p^{r}}+\alpha_{3 p^{r}}, \\
\phi_{4 p^{r}} \equiv \phi_{p^{r}}^{4}+6 \phi_{p^{r}}^{2} \alpha_{2 p^{r}}+4 \phi_{p^{r}} \alpha_{3 p^{r}}+3 \alpha_{2 p^{r}}^{2}+\alpha_{4 p^{r}},
\end{array}\right.
$$

and so on.

We note also that (2.3) implies

$$
\left\{\begin{array}{l}
\phi_{p^{r+1}} \equiv \phi_{p^{r}} \alpha_{1}+\alpha_{p^{r+1}}, \\
\phi_{2 p^{r+1}} \equiv \phi_{2 p^{r}} \alpha_{1}+2 \phi_{p^{r}} \alpha_{p^{r+1}}+\alpha_{2 p^{r+1}}, \\
\phi_{3 p^{r+1}} \equiv \phi_{3 p^{r}} \alpha_{1}+3 \phi_{2 p^{r}} \alpha_{p^{r+1}}+3 \phi_{p^{r}} \alpha_{2 p^{r+1}}+\alpha_{3 p^{r+1}} .
\end{array}\right.
$$

3. By means of (1.5) we can obtain certain congruences for the coefficient $A\left(k_{1}, k_{2}, k_{3}, \cdots\right)$. Indeed by (1.1) and (1.3)

$$
\begin{aligned}
& \phi_{n}\left(\phi_{p}, \alpha_{2 p}, \alpha_{3 p}, \cdots\right) \\
\equiv \sum A_{n}\left(k_{1}, k_{2}, k_{3}, \cdots\right)\left(\alpha_{1}^{p}+\alpha_{p}\right)^{k_{1}} \alpha_{2 p}^{k_{2}} \alpha_{3 p}^{k_{3}} \cdots \quad & (\bmod p),
\end{aligned}
$$

where the summation is over nonnegative $k_{j}$ such that

$$
k_{1}+2 k_{2}+3 k_{3}+\cdots=n \text {. }
$$

The right member of (3.1) is equal to

$$
\sum_{\left(k_{j}\right)} A_{n}\left(k_{1}, k_{2}, k_{3}, \cdots\right) \sum_{r=0}^{k_{1}}\left(\begin{array}{c}
k_{1} \\
r
\end{array}\right) \alpha_{1}^{p\left(k_{1}-r\right)} \alpha_{p}^{r} \alpha_{2 p}^{k_{2}} \alpha_{3 p}^{k_{3}} \cdots .
$$

On the other hand

$$
\phi_{p n}=\sum A_{p n}\left(h_{1}, h_{2}, h_{3}, \cdots\right) \alpha_{1}^{h_{1}} \alpha_{2}^{h_{2}} \alpha_{3}^{h_{3}} \cdots,
$$

summed over

$$
h_{1}+2 h_{2}+3 h_{3}+\cdots=p_{n} .
$$

It follows from (1.5) that

$$
A_{p n}\left(h_{1}, h_{2}, h_{3}, \cdots\right) \equiv 0
$$

except possibly when

$$
h_{j}=0 \quad(j>1, p+j) .
$$

When this condition is satisfied (3.4) becomes 


$$
h_{1}+p\left(h_{p}+2 h_{2 p}+\cdots\right)=p n ;
$$

consequently $h_{1}=p k_{1}$ and (3.3) becomes

$$
\phi_{p n} \equiv \sum A_{p n}\left(p k_{1}, 0, \cdots, 0, h_{p}, \cdots\right) \alpha_{1}^{p k_{1}} \alpha_{h}^{h p} \alpha_{2 p}^{h_{2 p} p} \cdots .
$$

We have therefore proved the following result:

THEOREM 1. The coefficient $A_{p n}\left(h_{1}, h_{2}, h_{3}, \cdots\right)$ occurring in (3.3) is certainly divisible by $p$ unless (3.5) is satisfied and $h_{1}=p k_{1}$. If these conditions are satisfied then

$$
A_{p n}\left(h_{1}, h_{2}, h_{3}, \cdots\right) \equiv\left(\begin{array}{l}
k_{1} \\
h_{p}
\end{array}\right) A_{n}\left(k_{1}-h_{p}, h_{p}, h_{2 p}, \cdots\right) \quad(\bmod p) .
$$

If we make use of (1.4) we obtain the following simpler

\section{TheOREM 2. Let}

$$
h_{1}+2 h_{2}+3 h_{3}+\cdots=p^{r} .
$$

Then the coefficient $A_{p r}\left(h_{1}, h_{2}, h_{3}, \cdots\right)$ is divisible by $p$ except when

$$
h_{i}=0 \quad(i \neq j), \quad h_{j}=p^{s},
$$

for some $j$, in which case

$$
A_{p^{r}}\left(h_{1}, h_{2}, h_{3}, \cdots\right) \equiv 1
$$

Using (2.10) and (2.11) we can obtain additional results. For example take

$$
h_{1}+2 h_{2}+3 h_{3}+\cdots=2 p^{r} .
$$

Then $A_{2 p^{r}}\left(h_{1}, h_{2}, h_{3}, \cdots\right)$ is divisible by $p$ unless (i) all $h_{s}=0(s \neq j)$, $h_{j}=1$ or 2 ; (ii) all $h_{s}=0(s \neq i, j), h_{i}=h_{j}=1$. In case (i) $A \equiv 1$, in case (ii) $A \equiv 2(\bmod p)$.

For $n=3 p^{r}$ the corresponding results are more complicated.

4. We turn now to the polynomial $C_{n}\left(\alpha_{1}, \alpha_{2}, \alpha_{3}, \cdots\right)$, the cycle indicator of the symmetric group [5, p. 68]:

$$
\begin{aligned}
C_{n} & =C_{n}\left(\alpha_{1}, \alpha_{2}, \alpha_{3}, \cdots\right)=\phi_{n}\left(\alpha_{1}, \alpha_{2}, 2 ! \alpha_{3}, \cdots\right) \\
& =\sum \frac{n !}{k_{1} ! k_{2} ! k_{3} \cdots}\left(\frac{\alpha_{1}}{1}\right)^{k_{1}}\left(\frac{\alpha_{2}}{2}\right)^{k_{2}}\left(\frac{\alpha_{3}}{3}\right)^{k_{3}} \cdots,
\end{aligned}
$$

where the summation is over all nonnegative $k_{j}$ such that

$$
k_{1}+2 k_{2}+3 k_{3}+\cdots=n .
$$

It is convenient to define $C_{0}=1$. 
Put

$$
c_{n}\left(k_{1}, k_{2}, k_{3}, \cdots\right)=\frac{n !}{k_{1} ! k_{2} ! k_{3} \cdots 1^{k_{1}} 2^{k_{2}} 3^{k_{3}} \cdots},
$$

the general coefficient of $C_{n}$. Clearly $c_{n}\left(k_{1}, k_{2}, k_{3}, \cdots\right)$ is integral and indeed a multiple of $A_{n}\left(k_{1}, k_{2}, k_{3}, \cdots\right)$.

From (4.1) we get the generating function

$$
G(t)=\sum_{n=0}^{\infty} G_{n} \frac{t^{n}}{n !}=\exp \left(\alpha_{1} t+\frac{1}{2} \alpha_{2} t^{2}+\frac{1}{3} \alpha_{3} t^{3}+\cdots\right) .
$$

For brevity put

$$
C(t)=\sum_{n=1}^{\infty} \frac{1}{n} \alpha_{n} t^{n}
$$

Differentiating (4.3) with respect to $t$ we get

$$
G^{\prime}(t)=C^{\prime}(t) G(t),
$$

that is

$$
\sum_{n=0}^{\infty} C_{n+1} \frac{t^{n}}{n !}=\sum_{r=0}^{\infty} \alpha_{r+1} t^{r} \sum_{n=0}^{\infty} C_{n} \frac{t^{n}}{n !}
$$

This implies

$$
C_{n+1}=\sum_{r=0}^{n} \frac{n !}{r !} \alpha_{n-r+1} C_{r},
$$

so that

$$
C_{n+1} \equiv \alpha_{1} C_{n}
$$

By repeated differentiation of (4.3) we get (compare (2.4))

$$
\frac{d^{p}}{d t^{p}} G(t) \equiv\left\{\left(C^{\prime}(t)\right)^{p}+C^{(p)}(t)\right\} G(t)
$$

Now since

$$
C^{\prime}(t)=\sum_{n=0}^{\infty} \alpha_{n+1} t^{n}, \quad C^{(p)}(t)=\sum_{n=0}^{\infty}(n+p-1) ! \alpha_{n+1} \frac{t^{n}}{n !},
$$

it is clear that

$$
\left(C^{\prime}(t)\right)^{p} \equiv \alpha_{1}^{p}, \quad C^{(p)}(t) \equiv-\alpha_{p}
$$

at the last step we have used Wilson's theorem. Thus (4.6) becomes 


$$
\sum_{n=0}^{\infty} C_{n+p} \frac{t^{n}}{n !} \equiv\left(\alpha_{1}^{p}-\alpha_{p}\right) \sum_{n=0}^{\infty} C_{n} \frac{t^{n}}{n !}
$$

so that

$$
C_{n+p} \equiv\left(\alpha_{1}^{p}-\alpha_{p}\right) C_{n}
$$

In particular we have

$$
C_{p} \equiv \alpha_{1}^{p}-\alpha_{p}
$$

and

$$
C_{n+r p} \equiv\left(\alpha_{1}^{p}-\alpha_{p}\right)^{r} C_{n}
$$

We remark that for $p=3,5,7,(4.8)$ is in agreement with the explicit values of $C_{n}$ given in [5, p. 69].

By (4.9) with $n=0$ we find that the coefficient

$$
c_{r p}\left(k_{1}, k_{2}, k_{3}, \cdots\right) \equiv 0
$$

unless all $k_{j}$ except $k_{1}$ and $k_{p}$ vanish and $k_{1}$ is a multiple of $p$; in this case we have

$$
c_{r p}\left(p k, 0, \cdots, 0, k_{p}, \cdots\right) \equiv(-1)^{k_{p}}\left(\begin{array}{l}
r \\
k
\end{array}\right) \quad(\bmod p)
$$

\section{REFERENCES}

1. P. Bachmann, Niedere Zahlentheorie, vol. 1, Leipzig, 1902.

2. E. T. Bell, Exponential polynomials, Annals of Math., 35 (1934), 258-277.

3. L. E. Dickson, Theorems on the residues of multinomial coefficients with respect to a. prime modulus, Quarterly J. Math., 33 (1902), 378-384.

4. A. Hurwitz, Über die Entwickelungscoeffizienten der lemniscatischen Functionen, Mathematische Werke, Basel, 2, (1933), 342-373.

5. J. Riordan, An Introduction to combinatorial analysis, New York, 1958.

6. J. Touchard, Propriétés arithmétiques de certains nombres recurrents, Ann. Soc. Sci. Bruxelles, A53 (1933), 21-31.

DUKE UNIVERSITY 


\section{PACIFIC JOURNAL OF MATHEMATICS}

\section{EDITORS}

\author{
RaLPh S. Phillips \\ Stanford University \\ Stanford, California \\ F. H. BRowNELL \\ University of Washington \\ Seattle 5 , Washington
}

A. L. Whiteman

University of Southern California

Los Angeles 7, California

L. J. Paige

University of California

Los Angeles 24, California

\author{
E. F. BECKENBACH \\ T. M. CHERRY
}

\author{
ASSOCIATE EDITORS

$\begin{array}{lll}\text { D. DERRY } & \text { H. L. ROYDEN } & \text { E. G. STRAUS } \\ \text { M. OHTSUKA } & \text { E. SPANIER } & \text { F. WOLF }\end{array}$

\section{SUPPORTING INSTITUTIONS}

\author{
UNIVERSITY OF BRITISH COLUMBIA \\ CALIFORNIA INSTITUTE OF TECHNOLOGY \\ UNIVERSITY OF CALIFORNIA \\ MONTANA STATE UNIVERSITY \\ UNIVERSITY OF NEVADA \\ NEW MEXICO STATE UNIVERSITY \\ OREGON STATE COLLEGE \\ UNIVERSITY OF OREGON \\ OSAKA UNIVERSITY \\ UNIVERSITY OF SOUTHERN CALIFORNIA
}

\author{
STANFORD UNIVERSITY \\ UNIVERSITY OF TOKYO \\ UNIVERSITY OF UTAH \\ WASHINGTON STATE COLLEGE \\ UNIVERSITY OF WASHINGTON \\ AMERICAN MATHEMATICAL SOCIETY \\ CALIFORNIA RESEARCH CORPORATION \\ HUGHES AIRCRAFT COMPANY \\ SPACE TECHNOLOGY LABORATORIES \\ NAVAL ORDNANCE TEST STATION
}

Mathematical papers intended for publication in the Pacific Journal of Mathematics should be typewritten (double spaced), and the author should keep a complete copy. Manuscripts may be sent to any one of the four editors. All other communications to the editors should be addressed to the managing editor, L. J. Paige at the University of California, Los Angeles 24, California.

50 reprints per author of each article are furnished free of charge; additional copies may be obtained at cost in multiples of 50 .

The Pacific Journal of Mathematics is published quarterly, in March, June, September, and December. The price per volume (4 numbers) is $\$ 12.00$; single issues, $\$ 3.50$. Back numbers are available. Special price to individual faculty members of supporting institutions and to individual members of the American Mathematical Society: $\$ 4.00$ per volume; single issues, $\$ 1.25$.

Subscriptions, orders for back numbers, and changes of address should be sent to Pacific Journal of Mathematics, 103 Highland Boulevard, Berkeley 8, California.

Printed at Kokusai Bunken Insatsusha (International Academic Printing Co., Ltd.), No. 6, 2-chome, Fujimi-cho, Chiyoda-ku, Tokyo, Japan.

\section{PUBLISHED BY PACIFIC JOURNAL OF MATHEMATICS, A NON-PROFIT CORPORATION}

The Supporting Institutions listed above contribute to the cost of publication of this Journal, but they are not owners or publishers and have no responsibility for its content or policies.

Reprinted 1966 in the United States of America 


\section{Pacific Journal of Mathematics}

\section{Vol. 11, No. 4}

A. V. Balakrishnan, Prediction theory for Markoff processes . . . . . . . . . . 1171

Dallas O. Banks, Upper bounds for the eigenvalues of some vibrating systems . . . . 1183

A. Białynicki-Birula, On the field of rational functions of algebraic groups ...... 1205

Thomas Andrew Brown, Simple paths on convex polyhedra .............. 1211

L. Carlitz, Some congruences for the Bell polynomials . . . . . . . . . . . . 1215

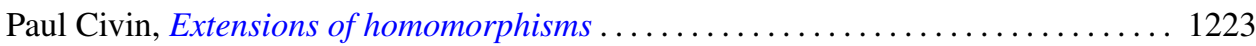

Paul Joseph Cohen and Milton Lees, Asymptotic decay of solutions of differential

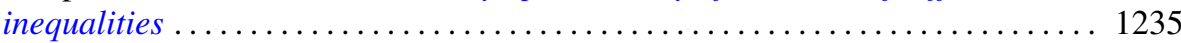

István Fáry, Self-intersection of a sphere on a complex quadric . . . . . . . . . . 1251

Walter Feit and John Griggs Thompson, Groups which have a faithful representation

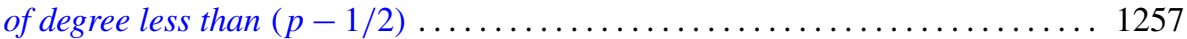

William James Firey, Mean cross-section measures of harmonic means of convex

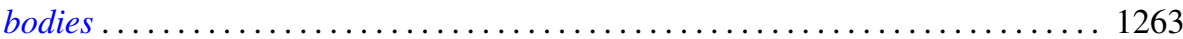

Avner Friedman, The wave equation for differential forms . . . . . . . . . . 1267

Bernard Russel Gelbaum and Jesus Gil De Lamadrid, Bases of tensor products of

Banach spaces ................................... 1281

Ronald Kay Getoor, Infinitely divisible probabilities on the hyperbolic plane . . . . 1287

Basil Gordon, Sequences in groups with distinct partial products . . . . . . . . . . . . 1309

Magnus R. Hestenes, Relative self-adjoint operators in Hilbert space . . . . . . . . . 1315

Fu Cheng Hsiang, On a theorem of Fejér ......................... 1359

John McCormick Irwin and Elbert A. Walker, On N-high subgroups of Abelian

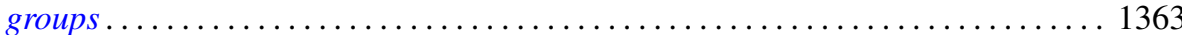

John McCormick Irwin, High subgroups of Abelian torsion groups . . . . . . . . . 1375

R. E. Johnson, Quotient rings of rings with zero singular ideal . . . . . . . . . . . 1385

David G. Kendall and John Leonard Mott, The asymptotic distribution of the time-to-escape for comets strongly bound to the solar system ...

Kurt Kreith, The spectrum of singular self-adjoint elliptic operators ....

Lionello Lombardi, The semicontinuity of the most general integral of the calculus of variations in non-parametric form ................................

Albert W. Marshall and Ingram Olkin, Game theoretic proof that Chebyshev inequalities are sharp

Wallace Smith Martindale, III, Primitive algebras with involution . . William H. Mills, Decomposition of holomorphs ..............

James Donald Monk, On the representation theory for cylindric algebras . . . . . . 1447

Shu-Teh Chen Moy, A note on generalizations of Shannon-McMillan theorem . . . . 1459

Donald Earl Myers, An imbedding space for Schwartz distributions . .

John R. Myhill, Category methods in recursion theory .........

Paul Adrian Nickel, On extremal properties for annular radial and circular slit mappings of bordered Riemann surfaces

Edward Scott O'Keefe, Primal clusters of two-element algebras . .

Nelson Onuchic, Applications of the topological method of Wazewski to certain

problems of asymptotic behavior in ordinary differential equations ...

Peter Perkins, A theorem on regular matrices................

Clinton M. Petty, Centroid surfaces .... 